\title{
RESEARCH HIGHLIGHT Revealing the secret behind Smo cholesterylation
}

\author{
Yuhong $\operatorname{Han}^{1}$ and Jin Jiang $\mathbb{D}^{1,2}$
}

(c) CEMCS, CAS 2022

Cell Research (2022) 32:327-328; https://doi.org/10.1038/s41422-022-00636-8

The GPCR family protein Smoothened (Smo) transduces Hedgehog signal across the plasma membrane. In a recent paper published in Cell Research, Hu et al. discovered that Smoothened undergoes an auto-cholesterylation process facilitated by calcium and demonstrated that covalent cholesterol attachment to Smo cysteine-rich domain is required for Hedgehog signal transduction.

Hedgehog $(\mathrm{Hh})$ signaling pathway governs embryonic development and adult tissue homeostasis across difference species. ${ }^{1}$ Smoothened (Smo), a class-F GPCR family member, serves as a hub in $\mathrm{Hh}$ signaling cascade by relaying the signal from the $\mathrm{Hh}$ receptor Patched (Ptc) to the Gli family of transcription factors. In resting cells, Ptc inhibits Smo in a substoichiometric manner, suggesting a catalytic mechanism rather than inhibition by direct protein-protein interaction. ${ }^{2}$ Together with the observation that Ptc is structurally related to the RND family of transporter, it has been postulated that Ptc functions as a small-molecule transporter to negatively regulate Smo and that this inhibition is alleviated upon ligand binding. Recent structural and biochemical studies have revealed that cholesterol and related oxysterols appear to be the long sought-after small molecules, and that cholesterol and related oxysterols bind both the $\mathrm{N}$-terminal cysteine-rich domain (CRD) and the seven transmembrane domains (7-TMs) of Smo to allosterically activate $\mathrm{Smo.}{ }^{3-7}$

In a previous study, Dr. Song et al. made an unexpected finding that Smo CRD is covalently attached by cholesterol at D95 in a manner stimulated by $\mathrm{Hh}$, and that mutating $\mathrm{D} 95$ to $\mathrm{N}$ blocked Smo cholesterylation and abolished Hh signaling; ${ }^{8}$ however, the underlying mechanism remains elusive. It is unclear whether a specific enzyme(s) is involved in Smo cholesterylation and how $\mathrm{Hh}$ stimulates this reaction. To address these issues, Song and colleagues carried out an extensive study and their tour de force was published recently in Cell Research. ${ }^{9}$ Most strikingly, they uncovered an auto-catalytic process responsible for Smo cholesterylation. In an in vitro reconstitution assay, the authors found that immunopurified Smo and its CRD but not the D95N mutant could be modified by cholesterol with a fast kinetics in the absence of additional cellular cofactors or energy supply, arguing against an enzymatic modification model. Because mutating Y130, which is spatially proximal to the cholesterol acceptor site D95, blocked Smo cholesterylation similarly to the D95N mutation, the authors hypothesized that D95 and Y130 might form an ester bond and that this high-energy intermediate could facilitate the final transfer of cholesterol to D95 (Fig. 1a). Indeed, mass spec analysis of purified Smo CRD provided evidence for such an intermediate, and theoretical calculation indicated that the transesterification reaction to cholesterol from the D95-Y130 ester bond is energetically favorable.

During the in vitro reconstitution experiments, the authors found that addition of calcium greatly facilitated Smo cholesterylation at a step after D95-Y130 ester bond formation (Fig. 1a). Because Smo cholesterylation is stimulated by $\mathrm{Hh}$, the authors asked whether $\mathrm{Hh}$ could promote Smo cholesterylation by increasing the local concentration of calcium in the subcellular compartment where Smo resides. They fused a calcium biosensor (GCaMP6s) to the N-terminus of Smo that also carried a mCherry fluorescent protein at its C-terminus and performed live imaging experiments. They found that GCaMP6s-Smo-mCherry resided mainly in early and recycling endosomes and that stimulating cells with $\mathrm{Hh}$ or a Smo agonist increased the luminal calcium level in Smo-localized endosomes. Furthermore, they found that the extracellular calcium served as the original source of calcium ion that is required for Smo cholesterylation. By CRISPR screening of a gRNA library targeting 39 distinct calcium channels, they identified ORAI1 as the major calcium channel responsible for the extracellular calcium influx into the cells. Additionally, two ORAI1-interacting proteins (STIM1 and STIM2) were found to be critical for Smo cholesterylation and Hh pathway activity. It is wellknown that STIM1/2-dependent ORAI1 activation is triggered by calcium depletion in the ER. Consistent with this, the authors observed that Hh stimulated a global reduction in ER calcium concentration. Using pharmacological inhibitors, they further delineated a Smo-Gai-IP $R$ pathway that mediates ER calcium depletion in response to $\mathrm{Hh}$. In addition, they found that $\mathrm{Hh}$ can stimulate Smo cholesterylation in cilia-depleted cells, implying that $\mathrm{Hh}$ induces Smo cholesterol modification outside the cilia. Taken together, these results suggest that $\mathrm{Hh}$ stimulates Smo cholesterylation by elevating the local calcium concentration in the Smo-localized endosomes through store-operated calcium entry.

Finally, the authors asked whether covalent modification of Smo CRD by cholesterol is essential for Smo activation. Because the D95N mutation used in the earlier study not only blocks Smo cholesterylation but also affects non-covalent cholesterol binding, one cannot distinguish whether loss of $\mathrm{Hh}$ signaling activity in Smo ${ }^{D 95 N / D 95 N}$ mutant embryos was due to the loss of Smo cholesterylation or simply due to the loss of non-covalent cholesterol binding. To obtain a definitive answer, the authors employed both biochemical and genetic approaches. First, they designed and synthesized several cholesterol analogs that can

\footnotetext{
${ }^{1}$ Department of Molecular Biology, UT Southwestern Medical Center, Dallas, TX, USA. ${ }^{2}$ Department of Pharmacology, UT Southwestern Medical Center, Dallas, TX, USA.

email: jin.jiang@utsouthwestern.edu
} 
a

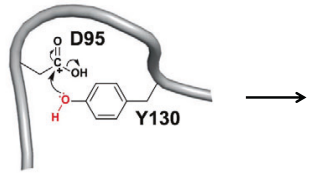

Smo CRD

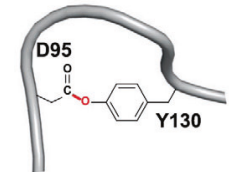

High-energy intermediate b - Hh

Smo

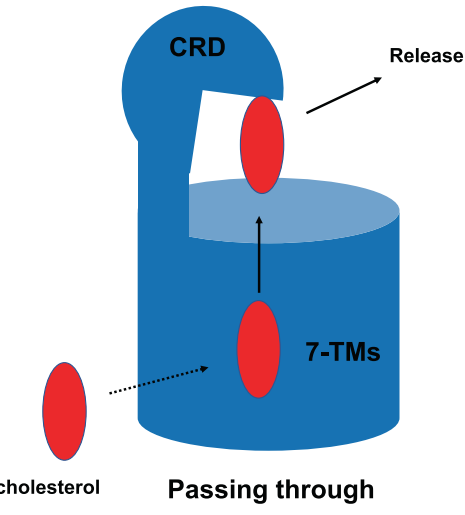

$+\mathrm{Hh}$

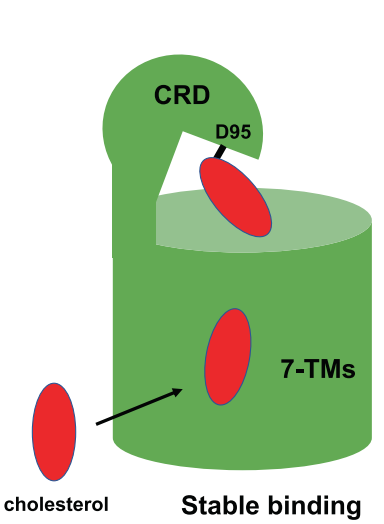

Fig. 1 Auto-cholesterylation of Smo is required for Smo activation. a Smo CRD forms a high-energy intermediate with an intramolecular ester bond formed between D95 and Y130. Calcium facilitates transesterification reaction to cholesterol from the D95-Y130 ester bond (adapted from Hu et al. 2022) ${ }^{9}$. b Smo 7-TMs form a cholesterol channel connected to its CRD. In the absence of Hh, Smo is less accessible to cholesterol. Low levels of cholesterol may enter the 7-TMs but are quickly transported out of the channel, which can be facilitated by transient binding of cholesterol to CRD. In the presence of $\mathrm{Hh}$, more cholesterol molecules enter the 7-TMs and covalent attachment of cholesterol to CRD may form a molecular "plug" to trap cholesterol inside the 7-TMs, leading to allosteric activation of Smo.

bind but are unable to covalently modify Smo CRD and found that these analogs failed to activate Smo. Second, they generated a D95E Smo variant $\left(\mathrm{Smo}^{\mathrm{D} 95 \mathrm{E}}\right)$ that could still bind cholesterol but failed to be covalently modified. Strikingly, Smo ${ }^{D 95 E / D 95 E}$ mouse embryos exhibited Hh signaling defects indistinguishable from $\mathrm{Smo}^{-/-}$embryos. Taken together, these complementary set of experiments firmly established that covalent cholesterol modification of Smo CRD is required for Hh signal transduction.

Although accumulating evidence suggests that cholesterol and related oxysterols are the endogenous ligands for Smo in recent years, the findings that cholesterol can be covalently attached to Smo through a calcium-regulated autocatalytic process provide novel insight into how cholesterol regulates Smo activation and Hh signaling. Like many other discoveries, this study also raises many questions, one of which is why Smo needs to be covalently modified by cholesterol for its activation. Considering a recent structural study suggesting that the 7-TMs of Smo may form a "cholesterol channel" connected to its $\mathrm{CRD}^{10}$ it is possible that transient binding of cholesterol to CRD may facilitate the passing of cholesterol through the channel whereas stable association of cholesterol with CRD bestowed by covalent modification may instead function as a molecular "plug" to trap the cholesterol bound to Smo 7-TMs (Fig. 1b), leading to allosteric activation of Smo and Hh signal transduction.

\section{REFERENCES}

1. Jiang, J. Semin. Cancer Biol. https://doi.org/10.1016/j.semcancer.2021.04.003 (2021).

2. Taipale, J., Cooper, M. K., Maiti, T. \& Beachy, P. A. Nature 418, 892-897 (2002).

3. Byrne, E. F. X. et al. Nature 535, 517-522 (2016).

4. Huang, P. et al. Cell 174, 312-324.e16 (2018).

5. Raleigh, D. R. et al. Mol. Cell 72, 316-327.e5 (2018).

6. Qi, X. et al. Nature 571, 279-283 (2019).

7. Deshpande, I. et al. Nature 571, 284-288 (2019).

8. Xiao, X. et al. Mol. Cell 66, 154-162.e10 (2017).

9. Hu, A. et al. Cell Res. https://doi.org/10.1038/s41422-022-00622-0 (2022).

10. Qi, X., Friedberg, L., De Bose-Boyd, R., Long, T. \& Li, X. Nat. Chem. Biol. 16, 1368-1375 (2020).

\section{ADDITIONAL INFORMATION}

Correspondence and requests for materials should be addressed to Jin Jiang.

Reprints and permission information is available at http://www.nature.com/ reprints 